\title{
ESTUDO RETROSPECTIVO DOS TRANSPLANTES DE CÓRNEA NO ESTADO DE ALAGOAS
}

\author{
Retrospective Study of Corneal Transplants in the State of Alagoas \\ Carolina Dourado Cardoso Tonhá', Andréa Maria Cavalcante Santos², Janaína Christina Norberto de Souza³, Marcella Cristina \\ Halliday Muniz ${ }^{4}$
}

\section{RESUMO}

Objetivo: Identificar o perfil dos pacientes submetidos a transplante de córnea no Estado de Alagoas, analisando sexo, idade e histórico oftalmológico. Avaliar as causas de indicações, técnicas cirúrgicas e complicações intra-operatórias dos pacientes transplantados. Método: Análise retrospectiva de 176 registros arquivados no Banco de Olhos do Estado de Alagoas, localizado no Hospital Universitário Prof. Alberto Antunes - Maceió/AL, no período de Outubro de 2004 a Dezembro de 2009. Resultados: Das 176 fichas analisadas, a idade variou de 27 dias de vida a 94 anos. Observou-se discreta prevalência do sexo feminino (51,7\%). A principal indicação para ceratoplastia penetrante foi ceratopatia bolhosa (31\%), seguida de leucoma, ceratocone e perfuração. Oitenta e um olhos apresentavam histórico cirúrgico prévio. Foram observados 13 casos de complicações intra-operatórias. Conclusão: Este estudo tornou possível o conhecimento do perfil dos pacientes transplantados em nosso Serviço. A média etária dos pacientes transplantados foi de 42 anos, sendo observada discreta prevalência entre o sexo feminino. A indicação óptica foi a mais frequente, e as cirurgias de caráter eletivo, na sua maioria. A principal indicação de ceratoplastia penetrante de córnea foi a ceratopatia bolhosa.

Descritores: Transplante de Córnea; Doenças da Córnea; Estudos Retrospectivos

Instituições:
${ }^{1}$ Fellow de Córnea e Doenças Externas na Santa Casa de São Paulo / São Paulo - SP - Brasil
${ }^{2}$ Diretora Médica do Banco de Tecidos do Hospital Universitário Alberto Antunes / UFAL-AL - Brasil
${ }^{3}$ Fellow de Retina no Hospital Oftalmológico de Sorocaba / Sorocaba - SP - Brasil
${ }^{4}$ Residente de Oftalmologia no Hospital Universitário Professor Alberto Antunes/UFAL - AL - Brasil

Correspondência:

Carolina Dourado Cardoso Tonhá

Rua Capote Valente, número 136 / 103 - CEP 05409-000 - São Paulo-SP, Brasil

Tel: (11) 87699161

E-mail: carolina_ufa|@yahoo.com.br

\section{INTRODUÇÃO}

As doenças da córnea destacam-se como a segunda causa de cegueira reversível no mundo, sendo responsáveis por incapacitar uma importante parcela da população jovem ativa, gerando danos sociais e econômicos à comunidade. ${ }^{1}$

A indicação de transplante de córnea, também chamado ceratoplastia penetrante, varia de acordo com a epidemiologia e a prática do Serviço oftalmológico responsável pela realização do procedimento. ${ }^{2-4} \mathrm{Nas}$ últimas décadas, observaram-se alterações nas indicações desse procedimento, decorrente principalmente do desenvolvimento de instrumentais e técnicas cirúrgicas que colaboraram positivamente para o sucesso e o prognóstico cirúrgico. ${ }^{5,6}$

Além do avanço nas técnicas cirúrgicas, o fato da córnea ser avascular e imunologicamente privilegiada, os métodos de preservação da córnea doadora e o manejo pós-operatório também são extremamente importantes para um resultado bem-sucedido, sendo a ceratoplastia penetrante o procedimento de maior sucesso entre os transplantes teciduais em humanos, e tem sido o mais realizado na atualidade. ${ }^{7,8}$

Classicamente, os transplantes de córnea podem ter finalidade óptica, terapêutica, tectônica e cosmética, que objetivam promover a visão, o controle da doença corneana refratária ao tratamento clínico, preservar a integridade do globo ocular e remover cicatrizes ou depósitos ceráticos, respectivamente. ${ }^{9,10}$ 
O Serviço de Oftalmologia do Hospital Universitário Professor Alberto Antunes realiza transplantes de córnea desde 2004.

Até o presente momento, pouco se conhece a respeito das ceratoplastias penetrantes de córnea realizadas nesta Instituição. Assim, este estudo reveste-se de importância epidemiológica, tendo como objetivo determinar o perfil dos pacientes transplantados, avaliando seu histórico oftalmológico e as principais indicações dos transplantes de córnea realizados.

\section{MÉTODOS}

Foram analisados retrospectivamente os registros arquivados no Banco de Olhos do Estado, localizado no Hospital Universitário Prof. Alberto Antunes - Maceió/AL, dos 176 pacientes submetidos à ceratoplastia penetrante no Estado de Alagoas, no período compreendido entre outubro de 2004 e dezembro de 2009.

Os resultados da análise foram anotados em um protocolo padronizado, no qual constavam além dos dados de identificação do paciente (idade e sexo), campos para preenchimento dos itens: doenças oculares pré-operatórias, tipo e propósito do procedimento cirúrgico realizado (se combinado com extração de catarata), ocorrência de complicações intra-operatórias e indicação do transplante.

A análise dos dados obtidos é exposta neste trabalho através de médias, desvio padrão e porcentagens.

O estudo obteve aprovação do Comitê de Ética em Pesquisa da Instituição onde foi realizado, sob número de protocolo 009981/2010-81.

\section{RESULTADOS}

Dos 176 pacientes transplantados, $85(48,29 \%)$ eram do sexo masculino e $91(51,70 \%)$ do sexo feminino. A idade da amostra estudada variou de 27 dias de vida a 94 anos, sendo a média de 42,26 anos. (Figura 1)

Figura 1 - Faixas etárias dos pacientes submetidos a transplante de córnea no Estado de Alagoas, no período de outubro de 2004 a dezembro de 2009

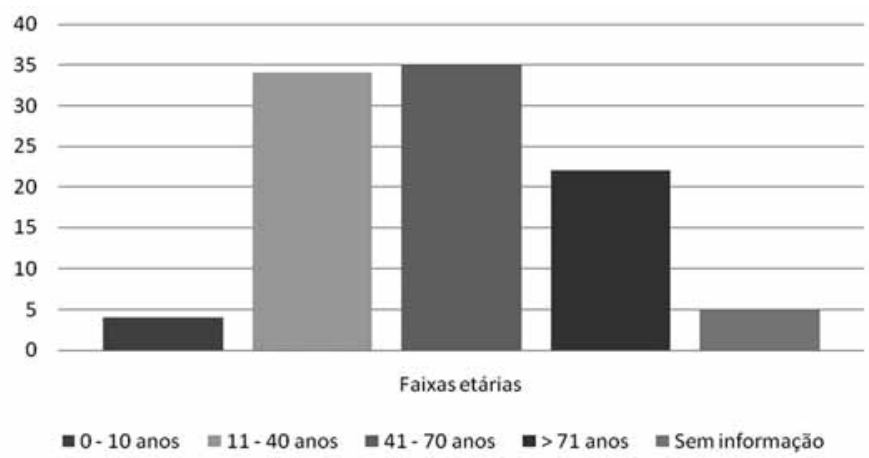

Dentre as doenças oculares pré-operatórias, 30 olhos $(17,4 \%)$ apresentavam glaucoma e $81(46,02 \%)$ já haviam sido submetidos a algum tipo de intervenção cirúrgica. Ressalta-se ainda que cerca de 47 olhos $(26,70 \%)$ eram pseudofácicos antes do procedimento.
Quanto à caracterização do procedimento cirúrgico, observou-se que foram realizados $35(20,14 \%)$ procedimentos combinados com extração de catarata, sendo que em 28 destes (80\%) implantouse lente intra-ocular (cirurgia tríplice). Segundo o propósito da ceratoplastia, foi observado que a grande maioria teve finalidade óptica (86\%), seguido de tectônica (12\%), cosmética (1\%) e, por fim, terapêutica (1\%). No que se refere ao tipo de cirurgia, $85 \%$ foram cirurgias eletivas e 15\% de emergência. (Figura 2)

Figura 2 - Finalidades dos transplantes de córnea realizados no Estado de Alagoas, no período de outubro de 2004 a dezembro de 2009.

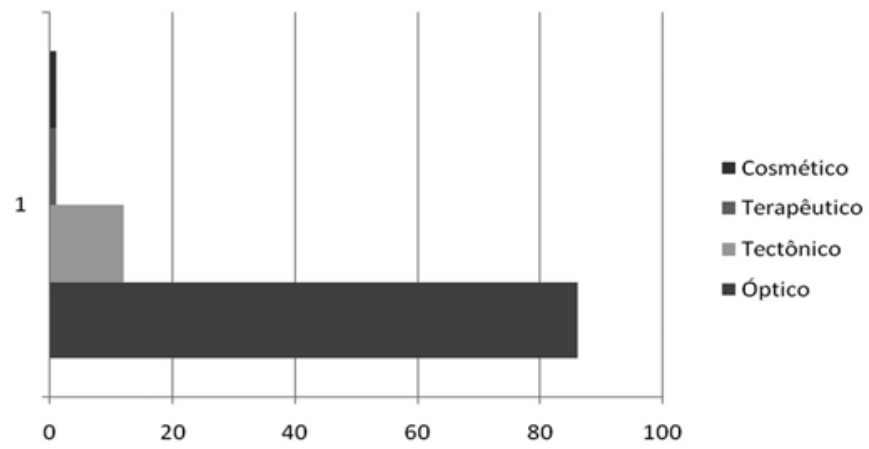

Foram evidenciados 13 casos (7,38\%) de complicações intraoperatórias durante a ceratoplastia penetrante, dentre eles: expulsão espontânea do cristalino opaco, aumento da pressão intravítrea por elevação da pressão arterial, afacia, presença de nova descemetocele, hipotonia, hemorragia iriana e ruptura da cápsula posterior do cristalino.

A principal causa de indicação de ceratoplastia penetrante foi ceratopatia bolhosa (31\% dos casos), seguida por leucoma (17\%), ceratocone (15\%), e perfuração (11\%). Dentre o grupo dos leucomas, o congênito foi o responsável por $86 \%$ dos casos de ceratoplastia penetrante em menores de 10 anos. (Tabela 1)

Tabela 1 - Indicações dos transplantes de córnea realizados no Estado de Alagoas, no período de outubro de 2004 a dezembro de 2009

\begin{tabular}{|l|c|}
\hline Indicações & $\%$ \\
\hline Ceratopatia bolhosa & 31 \\
\hline Perfuração corneana & 11 \\
\hline Leucoma & 17 \\
\hline Ceratocone & 15 \\
\hline Falência primária & 1 \\
\hline Rejeição & 2 \\
\hline Trauma & 1 \\
\hline Degenerações/Distrofias & 10 \\
\hline Outras causas & 4 \\
\hline Sem informação & 8 \\
\hline
\end{tabular}

\section{DISCUSSÃO}

A idade da amostra estudada variou de 27 dias a 94 anos, comparável a outro estudo prospectivo publicado, que apresentou 
faixa etária dos pacientes transplantados entre $0-88$ anos. ${ }^{11}$

Um estudo relatou que $52,2 \%$ dos pacientes submetidos a transplante de córnea em sua amostra eram do sexo feminino, ${ }^{3}$ dado semelhante ao apresentado neste estudo, onde o sexo feminino figura com $52 \%$ dos pacientes.

Nesta avaliação, a prevalência do procedimento cirúrgico tríplice em $19,88 \%$ coincide com os dados da literatura pesquisada, que registra tal procedimento como responsável por $20 \%$ das cirurgias de transplante penetrante de córnea. ${ }^{10,12}$

Dentre as complicações observadas no intra-operatório, ressalta-se a ocorrência de um caso de ruptura da cápsula posterior do cristalino em um procedimento combinado $(2,85 \%)$. Outros estudos revelam incidência semelhante, o que reforça a íntima relação da ocorrência de determinadas complicações com a experiência do cirurgião. ${ }^{7,10}$

A ceratopatia bolhosa figurou como a principal indicação de ceratoplastia penetrante, correspondendo a 31\% dos casos, resultado semelhante ao encontrado em outros estudos, ${ }^{4,13-17}$ Dentre os casos de ceratopatia bolhosa, a grande maioria (67\%) dos olhos eram pseudofácicos.

A segunda indicação mais frequente foi leucoma, em 17\% dos casos. Em estudos envolvendo apenas crianças submetidas a transplante penetrante de córnea, a maioria dos casos foi por opacidade congênita, seguida de opacidade adquirida, corroborando este estudo que $86 \%$ das causas de indicação de transplante de córnea entre os menores de 10 anos foi leucoma congênito. ${ }^{10,18}$

A terceira indicação foi ceratocone em $15 \%$ dos casos, dado que se assemelha ao da literatura pesquisada, em que esta predominou entre a segunda e terceira causas de indicação de transplante, variando de 11,4 a $20,7 \%$ do total. ${ }^{7,8,18,19}$ O ceratocone, no entanto, figura como a principal indicação de transplante de córnea na
América Latina e Europa, como afirmam outros estudos. ${ }^{3,4,13,16}$

A quarta indicação mais frequente neste estudo foi perfuração corneana consequente a processo infeccioso, respondendo por $11 \%$ dos casos. Este dado justifica a elevada porcentagem de transplante de córnea tectônico (12\%), em detrimento da indicação terapêutica (1\%) na amostra estudada, tendo em vista que o primeiro tem por princípio restaurar a integridade estrutural da córnea e do globo ocular diante de uma perfuração iminente ou já ocorrida. Sabe-se que as perfurações corneanas podem resultar de uma grande variedade de desordens além de infecções, destacandose inflamações e traumas e têm potencial de ocasionar graves sequelas visuais. ${ }^{10}$ Essa prevalência elevada pode ser explicada pelas características epidemiológicas do Estado de Alagoas, que possui um dos menores índices de desenvolvimento humano do país e situa-se entre os mais pobres da região nordeste. Tal fato salienta a dificuldade de acesso da população a serviços de saúde, além da dificuldade na manutenção de tratamentos custosos necessários na maioria dos casos de ceratites infecciosas.

\section{CONCLUSÕES}

Este estudo tornou possível o conhecimento do perfil dos pacientes transplantados em nosso Serviço, demonstrando a relevância de se conhecer o perfil da população, no que tange o manejo clínico adequado.

Identificou-se que a média etária dos pacientes transplantados foi de 42 anos, sendo observada discreta prevalência do sexo feminino. As cirurgias realizadas tiveram, predominantemente, propósito óptico e foram de caráter eletivo em sua grande maioria. A principal indicação de transplante penetrante de córnea foi ceratopatia bolhosa seguida de leucoma, ceratocone e perfuração.

\section{ABSTRACT}

Purpose: To identify profile of patients undergoing corneal transplantation in the State of Alagoas, analyzing the gender, age and ophthalmic history. To assess the reasons for the indications, surgical techniques and intraoperative complications of patients. Methods: Retrospective analysis of 176 medical records at the Eye Bank of the State of Alagoas located in the University Hospital Alberto Antunes - Maceió / AL along the October 2004 to December 2009 period. Results: 176 records were analyzed, and the age of the sampling ranged from 27 days old to 94 years old. There was a slight prevalence of females (51.7\%). Main indication to penetrating keratoplasty was bullous keratopathy (31\%), followed by leucoma, keratoconus and cornea perforation. Eighty-one eyes had previous surgical history. There were 13 cases of intraoperative complications. Conclusions: This study has allowed understanding the profile of transplant patients in our hospital. The mean age of transplanted patients was 42 years, with slightly higher prevalence of females. Visual indication was the most common, and mostly of elective surgeries. The main indication for penetrating keratoplasty was bullous keratopathy.

Keywords: Corneal Transplantation; Corneal Diseases; Retrospectives Studies

\section{REFERÊNCIAS}

1. Adán CBD, Diniz AR, Periatto D, Hirai FE, Sato EH. Dez anos de doação de córneas no Banco de Olhos do Hospital São Paulo: perfil dos doadores de 1996 a 2005. Arq Bras Oftalmol. 2008;71(2):176-81.

2. Urbano AP, Akaishi PMS, Sarmento S, Urbano AP, Costa VP, Arieta CL. Evolução das ceratoplastias penetrantes realizadas por residentes. Rev Bras Oftalmol. 2001;60:741-8.

3. Dantas MCN, Dantas PEC, Holzchuh N, Lui Neto A, Giovedi Filho R, Giovedi M, et al. Indicações de transplante penetrante de córnea: 1991-1995. Arq Bras Oftamol. 1998;61:26-33.

4. Endriss D, Cunha F, Ribeiro MP, Toscano J. Ceratoplastias penetrantes realizadas na Fundação Altino Ventura: revisão dos resultados e complicações. Arq Bras Oftalmol. 2003;66(3):273-7.

5. Flowers CW, Chanq KY, McLeod SD, Irvine JA, McDonnell PJ, Rao N, et al. Changing indications for penetrating keratoplasty, 1989-1993. Cornea. 1995;14(6):583-8. 
6. Sano FT, Dantas PEC, Silvino WR, Sanchez JZ, Sano RY, Adams F, ET al. Tendência de mudança nas indicações de transplante penetrante de córnea. Arq Bras Oftalmol. 2008;71(3): 400-4.

7. Dobbins KR, Price FW Jr, Whitson WE. Trends in the indications for penetrating keratoplasty in the midwestern United States. Cornea. 2000;19(6):813-6.

8. Inoue K, Amano S, Oshika T, Sawa M, Tsuru T. A 10-year review of penetrating keratoplasty. Jpn J Ophthalmol. 2000;44(2):139-45.

9. Aiken-O'Neill P, Mannis MJ. Summary of corneal transplant activity Eye Bank Association of America. Cornea. 2002;21(1):1-3.

10. Krachmer JH, Mannis MJ, Holland EJ. Cornea: Surgery of the Cornea and the Conjunctiva. 2nd ed. St Louis: Mosby; 2005.

11. Flores VGC, Dias HLR, Castro RS. Indicações para ceratoplastia penetrante no Hospital das Clínicas - UNICAMP. Arq Bras Oftalmol. 2007;70(3):505-8.

12. Sridhar MS, Murthy S, Bansal AK, Rao GN: Corneal triple procedure: indications, complications and outcomes: a developing country scenario. Cornea 2000;19:333-5

13. Cattani S, Kwitko S, Kroeff MAH, Marinho D, Rymer S, Bocaccio FL. Indicações de transplante de córnea no Hospital das Clínicas de Porto Alegre. Arq Bras Oftalmol. 2002;65:95-8.

14. Cosar CB, Sridhar MS, Cohen EJ, Held EL, Alvim PT, Rapuano CJ, et al. Indications for penetrating keratoplasty and associated procedures, 1996-2000. Cornea. 2002;21(2):148-51.

15. Randleman JB, Song CD, Palay DA. Indications for and outcomes of penetrating keratoplasty performed by resident surgeons. Am J Ophthalmol. 2003;136(1):68-75.

16. Ávila MP, Sugano DM, Póla CM, Lima VL, Rehder JRCL. Estatística do Banco de Olhos do ABC: 1997-2000. Arq Med ABC. 2001;24(1):56-9.

17. Kang PC, Klintworth GK, Kim T, Carlson AN, Adelman R, Stinnett S, et al. Trends in the indications for penetrating keratoplasty, 1980-2001. Cornea. 2005;24(7):801-3.

18. Cowden JW: Penetrating Keratoplasty in infants and children. Ophthalmology 1990;97:324-9.

19. Al-Yousuf N, Mavrikakis I, Mavrikakis E, Daya SM. Penetrating keratoplasty: indications over a 10 year period. Br J Ophthalmol. 2004;88(8):998-1001. 